\title{
One-dimensional soil temperature assimilation experiment based on unscented particle filter and Common Land Model
}

\author{
Xiao Lei FU ${ }^{1}$, Bao Ming JIN ${ }^{1}$, Xiao Lei JIANG ${ }^{2}$, Cheng CHEN ${ }^{1}$ \\ ${ }^{1}$ College of Civil Engineering, Fuzhou University, Fuzhou 350116, China \\ ${ }^{2}$ State Key Laboratory of Hydrology-Water Resources and Hydraulic Engineering, Hohai University, Nanjing 210098, China
}

\begin{abstract}
Data assimilation is an efficient way to improve the simulation/prediction accuracy in many fields of geosciences especially in meteorological and hydrological applications. This study takes unscented particle filter (UPF) as an example to test its performance at different two probability distribution, Gaussian and Uniform distributions with two different assimilation frequencies experiments (1) assimilating hourly in situ soil surface temperature, (2) assimilating the original Moderate Resolution Imaging Spectroradiometer (MODIS) Land Surface Temperature (LST) once per day. The numerical experiment results show that the filter performs better when increasing the assimilation frequency. In addition, UPF is efficient for improving the soil variables (e.g., soil temperature) simulation/prediction accuracy, though it is not sensitive to the probability distribution for observation error in soil temperature assimilation.
\end{abstract}

\section{Introduction}

Soil temperature is an important variable in land surface processes, as it not only influences the energy partitioning [1-2], but also affects the water cycle of land-atmosphere system [3-4]. However, it is difficult to obtain accurate soil temperature information at different soil depths at large scales through in situ observation, land surface model, or remote sensing. It is impractical to conduct intensive in situ observations over large areas although its accuracy is high [5]. Using a land surface model, one can simulate soil temperature at the large scales even for different soil layers, but the accuracy of such simulation is variable and can be quite low [6-7]. Through satellite remote sensing, one can derive soil surface temperature information at continental and global scales, but satellite retrieval of soil temperature is also limited to the soil surface at a relative large sacale, and is not error free [8-10].

Data assimilation techniques were introduced to geosciences such as weather forecasting and hydrology as an efficient way to incorporate observations into model states to improve the accuracy of simulated state variables [11-13]. In the past few decades, numerous data assimilation methods have been developed for different applications [5,14]. For example, Evensen (1994) proposed ensemble Kalman filter to solve the high-order nonlinear problems, and has been used in hydrologic applications [15]. Van der Merwe [16] introduced unscented particle filter, and its performance was tested by Han and $\mathrm{Li}$ [17] through soil temperature assimilation experiment. Yu et al $[2,5]$ introduced ensemble particle filter, compared it to ensemble Kalman filter and particle filter through soil temperature simulation, and used it to improve the soil temperature profile by assimilating MODIS LST. This study takes unscented particle filter as an example, and aims to test its performance with different assimilation frequencies and probability distributions of observation error in land surface state application.

\section{Study area and data description}

Walnut Gulch Experimental Watershed (WGEW), located in southeastern Arizona and with an area of approximately $149 \mathrm{~km}^{2}$ [18], is selected as the study area (Fig. 1), given the large amount of in situ and remote sensing data available in the watershed. The data used in the study include the meteorological forcing data, soil hydrological properties, and MODIS LST. Detailed information about the study area can be found in Keefer et al. [18], hence not repeated here. The observed data in KENMet site are used to evaluate the filter performance.

\section{Methodology}

\subsection{Model operator}

The model used in this study is the Common Land Model (CLM), and details about CLM can be found in Oleson et al. [19]. The CLM can have up to 10 layers in the soil column and up to five layers of snow depending on the snow depth [19]. In this study, the soil column is discretized into five layers. Only the one-dimensional

*Corresponding author: Email: fuxiaolei518@163.com 
heat diffusion equation for soil temperature in CLM describes here briefly as follows:

$c \frac{\partial T}{\partial t}=-\frac{\partial F}{\partial z}$

$F=-\lambda \frac{\partial T}{\partial z}$

Where $c$ is volumetric soil heat capacity $\left(\mathrm{Jm}^{-3} \mathrm{~K}^{-1}\right)$, $T$ is soil temperature $(K), t$ is time $(h), F$ is heat flux $\left(W m^{-2}\right), \lambda$ is thermal conductivity $\left(W m^{-1} K^{-1}\right)$, and $z$ is vertical depth $(m)$.

\subsection{Unscented particle filter (UPF)}

The unscented particle filter is proposed by Van der Merwe [16], using the unscented Kalman filter (UKF, which can be found in Han and Li [17]) to obtain the mean and covariance of the proposal distribution for each particle. The details of UPF can be found in Han and Li [17] and not list in this study to avoid redundancy.

In addition, to evaluate the performance of the filter, the root mean square error (RMSE) is used to be the only evaluation metric for soil temperature.

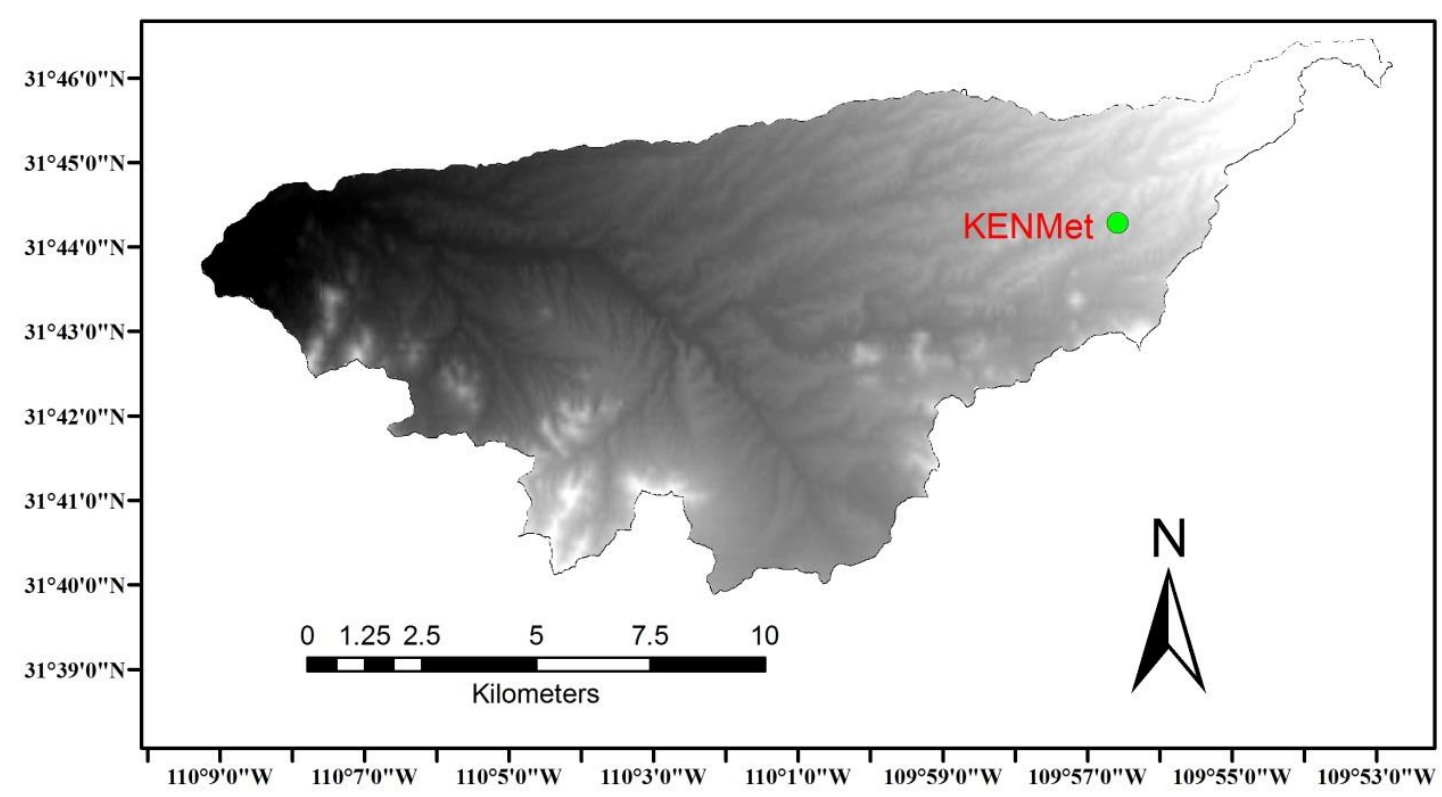

Fig. 1. The location of KENMet site which is selected to verify the filters performance at WGEW.

\section{Results and discussions}

In the numerical experiments, the observed soil surface temperature, MODIS LST from 2 October (day 276) to 21 October (day 295), 2004 were assimilated into CLM. To show the comparison results clearly, the last 5-day (from day 286 to day 290) simulated and assimilated results are used to verify the performance of the assimilation methods.

\subsection{Assimilating hourly in situ soil surface layer temperature into the model}

The RMSE between the simulated/assimilated results and observed data in KENMet site of the 5-day is shown in table 1. From the table, it is noted that UPF performs well for improving soil temperature profile except the last soil layer. In addition, it can be found that the filter is not sensitive to the probability distribution in soil temperature simulation. Therefore, only the average simulated and assimilated results of the 5-day with Gaussian distribution for hourly observations assimilation shown in Fig. 2.

From Fig. 2, it is noted that the averaged open loop (simulated results without assimilation) is far from the observed data at each layer, especially the open loop underestimated soil temperature significantly when soil temperature reached to its peak value for the first three layers. The reason may be attributed to the uncertainty of input and model.

Fig. 2 shows that the assimilated results of the filter are closer to the observed data than the simulated data in the first four layers. But for the fifth layer (e.g., the bottom layer), the performance of the filter is poor, this may be because only the soil surface temperature was assimilated, the soil heat flux is assumed to zero at the under boundary in the model and soil temperature at this layer changes in-obvious. The figure shows that the assimilated results underestimated the soil temperature at some time as the open loop underestimated it at the same time. However, the filter performed much better when soil temperature reached to its peak value for the first three layers. In addition, it is noted that the amplitude of the simulated/assimilated soil temperature is smaller than observed at these layers. 
Table 1. The 5-day soil temperature RMSE (K) of simulation and UPF.

\begin{tabular}{|c|c|c|c|c|c|}
\hline Depth (cm) & Simulation & Hourly Gaussian & Hourly uniform & LST Gaussian & LST uniform \\
\hline 0 & 2.2024 & 1.5575 & 1.5396 & 2.0537 & 2.0763 \\
\hline 5 & 1.6029 & 1.0079 & 1.0038 & 1.1670 & 1.1774 \\
\hline 15 & 0.9673 & 0.6585 & 0.6504 & 0.8619 & 0.8857 \\
\hline 50 & 0.4277 & 0.2810 & 0.3019 & 0.2820 & 0.3127 \\
\hline 100 & 0.1801 & 0.2597 & 0.3134 & 0.3846 & 0.3701 \\
\hline
\end{tabular}

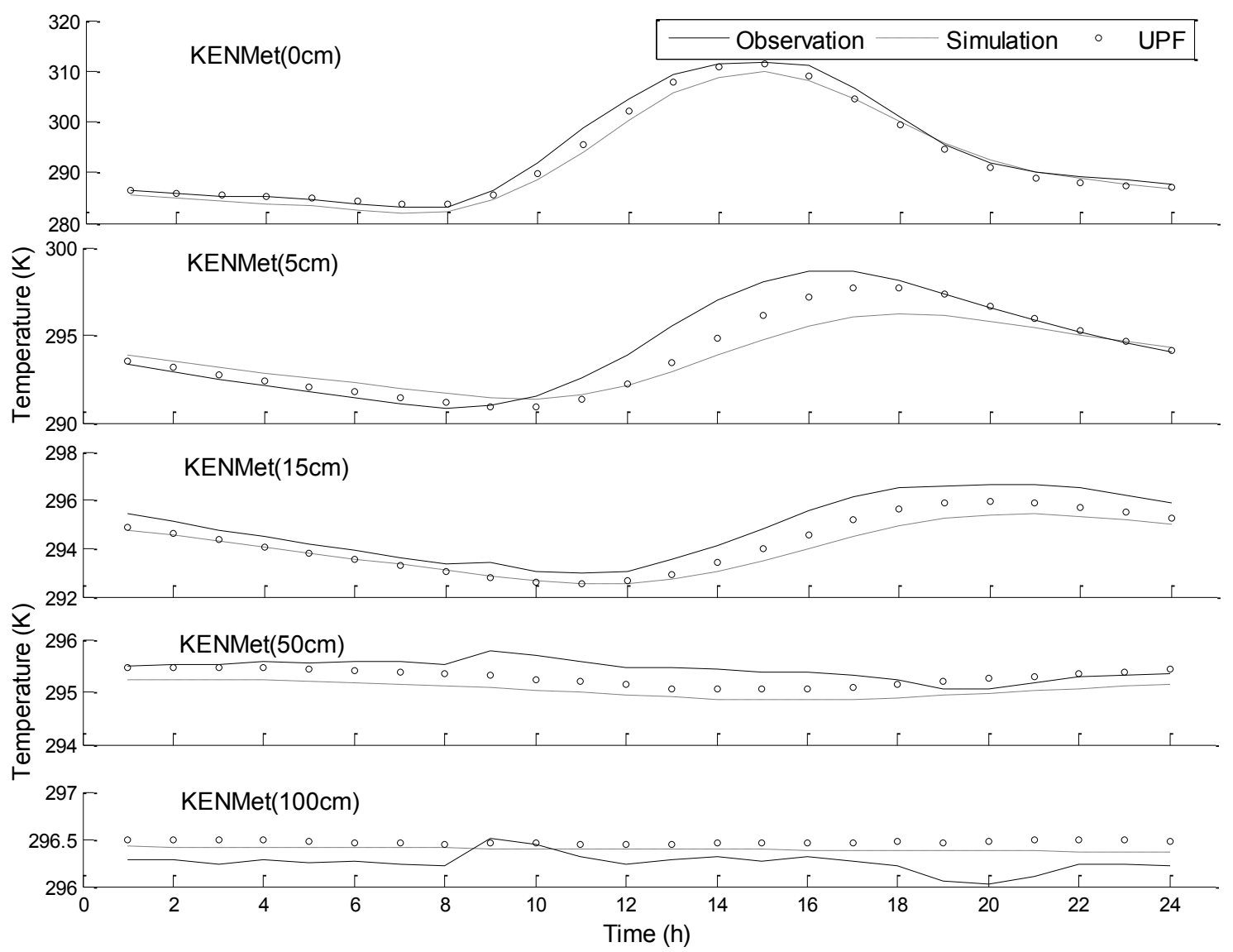

Fig. 2. The 5-day average results of assimilating hourly in situ soil surface observations using EnKF and UPF with Gaussian distribution.

\subsection{Assimilating the original MODIS LST once per day}

In this experiment, we assimilate the original MODIS LST into the model once per day as there is only one MODIS LST of the WGEW every day when the MODIS sensor passes over the area about from 10:30am to 11:00am. Compared with assimilation of hourly in situ observations in section 4.1, the assimilation frequency in this experiment is $1 / 24$ of that in above experiment. Similarly, the 5-day (from day 286 to day 290) results are also used to verify the filter performance.

Similarly, only the average results of the 5-day with Gaussian distribution for MODIS LST assimilation are shown in Fig. 3. The results in this figure are similar to that in Fig. 2. However, it is noted that the filter assimilated results are closer to the observed data at the first four layers in Fig. 3, but not significant like that in Fig. 2. The filter performs poorly in the fifth layer, which is consistent to the results in section 4.1.

Comparing the assimilating hourly in situ soil surface temperature and assimilating MODIS LST experiments, it is noted that the main difference of the two experiments is the assimilation frequency, and the filter performs better with increasing the assimilated observations. Comparing the experiment with Gaussian distribution to that with uniform distribution for observation, it can be found that the difference of the filter performance is small. This demonstrates that the filter is not sensitive to error distribution for observation in some state variables (e.g., soil temperature) simulation without considering the assimilation frequency, this may be because the variables are relative stable and not varied sharply in spatiotemporal. 


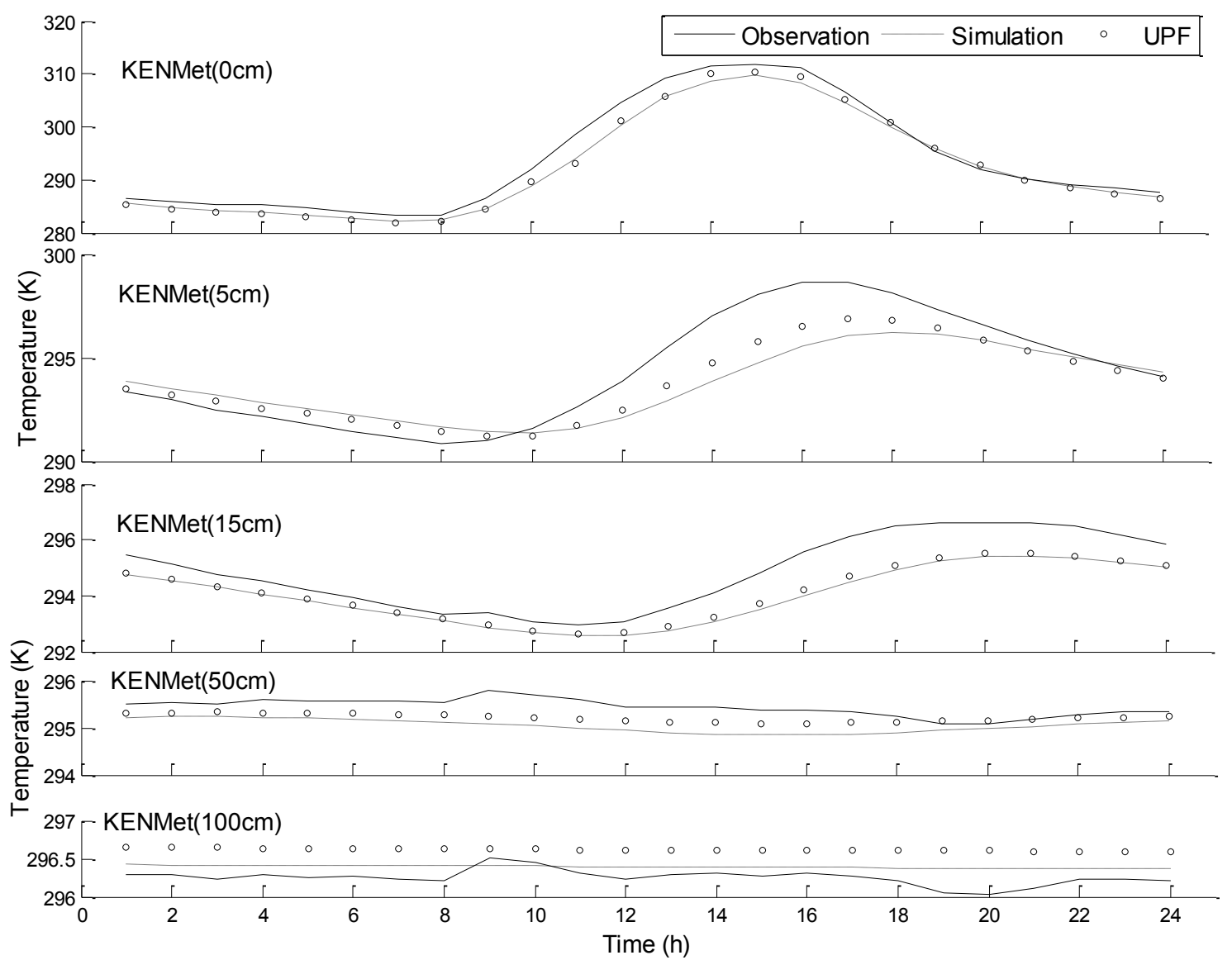

Fig. 3. The 5-day average results of assimilating MODIS LST using EnKF and UPF with Gaussian distribution. The MODIS sensor passes over the area about from 10:30am to 11:00am.

\section{Conclusion}

In this study, we evaluate UPF performance based on different assimilation frequencies and different probability distributions of observation error through soil temperature simulation at Walnut Gulch Experimental Watershed (WGEW) in Arizona, United States. The numerical experiment shows that the filter performs well in soil temperature assimilation at upper several soil layers. When assimilation frequency is low (e.g., once per day), it can improve the simulation accuracy, although the improved accuracy is not significant. Furthermore, it performs well significantly as increased the assimilation frequency (e.g., once per day to hourly). In addition, the filter is not sensitive to error distribution for observation in soil temperature assimilation without considering assimilation frequency.

\section{References}

1. Yu Z, Carlson T N, Barron E J, et al. On evaluating the spatial-temporal variation of soil moisture in the Susquehanna River Basin [J]. Water Resources Research, 2001, 34: 1313-1326.

2. $\mathrm{Yu} \mathrm{Z,} \mathrm{Fu} \mathrm{X,} \mathrm{Lü} \mathrm{H,} \mathrm{et} \mathrm{al.} \mathrm{Evaluating} \mathrm{ensemble}$ Kalman, particle and ensemble particle filters through soil temperature prediction $[\mathrm{J}]$. Journal of
Hyrologic Engineering, 2014, 19: 04014027.

3. Yu Z, Barron E J, Yarnal B, et al. Evaluation of basin scale hydrologic response to a multi storm simulation [J]. Journal of Hydrology, 2002, 257: 212-225.

4. Huang $\mathrm{C}, \mathrm{Li} \mathrm{X}, \mathrm{Lu} \mathrm{L}$. Retrieving soil temperature profile by assimilating MODIS LST products with ensemble Kalman filter [J]. Remote Sensing of Environment, 2008, 112(4): 1320-1336.

5. $\mathrm{Yu} \mathrm{Z}, \mathrm{Fu} \mathrm{X}$, Luo L. One-dimensional soil temperature simulation with Common Land Model by assimilating in situ observations and MODIS LST with the ensemble particle filter [J]. Water Resources Research, 2014, 50: 6950-6965.

6. Mihalakakou G. On estimating soil surface temperature profiles [J]. Energy and Buildings, 2002, 34(3): 251-259.

7. Wu C L, Chau K W, Huang J S. Modeling coupled water and heat transport in a soil-mulch-plant-atmosphere continuum (SMPAC) system [J]. Applied Mathematical Modeling, 2007, 31 (2): 152-169.

8. Wan Z. Dozier J. A generalized split-window algorithm for retrieving land-surface temperature from space [J]. IEEE Transactions on Geoscience and Remote Sensing, 1996, 34(4): 892-905.

9. Njoku E G, Li L. Retrieval of land surface parameters using passive microwave measurements 
at $6-18 \mathrm{GHz}[\mathrm{J}]$. IEEE Transactions on Geoscience and Remote Sensing, 1999, 37(1): 79-93.

10. Owe M, Van de Griend A A. On the relationship between thermodynamic surface temperature and high frequency $(37 \mathrm{GHz})$ vertical polarization brightness temperature under semi-arid conditions [J]. International Journal of Remote Sensing, 2001, 22(17): 3521-3532.

11. Kumar S V, Reichle R H, Peters-Lidard C D, et al. A land surface data assimilation framework using the land information system: Description and applications [J]. dvances in Water Resources, 2008, 31(11): 1419-1432.

12. McLaughlin D. An integrated approach to hydrologic data assimilation: interpolation, smoothing, and filtering $[\mathrm{J}]$. Advances in Water Resources, 2002, 25(8-12):1275-1286.

13. Reichle R H. Data assimilation methods in the earth sciences [J]. Advances in Water Resources, 2008, 31(11): 1411-1418.

14. Fu X, Yu Z, Luo L, et al. Investigating soil moisture sensitivity to precipitation and evapotranspiration errors using SiB2 model and ensemble Kalman filter
[J]. Stochastic Environmental Research and Risk Assessment, 2014, 28: 681-693.

15. McLaughlin D. Recent developments in hydrologic data assimilation [J]. Reviews of Geophysics, 1995, 33: 977-984.

16. Van der Merwe R. Sigma-Point Kalman Filters for Probalistic Inference in Dynamic State-Space Models [D]. Oregon Health and Science University, 2004.

17. Han $\mathrm{X}, \mathrm{Li} X$. An evaluation of the nonlinear/non-Gaussian filters for the sequential data assimilation [J]. Remote Sensing of Environment, 2008, 112(4): 1434-1449.

18. Keefer T O, Moran M S, Paige G B. Long-term meteorological and soil hydrology database, Walnut Gulch Experimental Watershed, Arizona, United States [J]. Water Resources Research, 2008, 44: W05S07.

19. Oleson K, Dai Y, Bonan G, et al. Technical description of the Community Land Model (CLM) [M]. NCAR Technical Note NCAR/TN-461+STR, National Center for Atmospheric Research, Boulder, Colo, 2004. 